\title{
Relationships of the Psychological Influence of Food and Barriers to Lifestyle Change to Weight and Utilization of Online Weight Loss Tools
}

\author{
Martin Binks", Trevor van Mierlo and Christopher L Edwards
}

Binks Behavioral Health PLLC, 2801 Canter Drive, Hillsborough, NC 27278, USA

\begin{abstract}
Introduction: The psychological influence of food (PFS) and perceived barriers to lifestyle change (PBLC) were considered as predictors of body mass index and website tool utilization (TU) in an online weight loss program.

Materials and Methodology: An archival analysis of all $(\mathrm{N}=1361)$ overweight $/ \mathrm{obese}\left(\mathrm{BMI} \mathrm{M}=31.6 \pm 6.24 \mathrm{~kg} / \mathrm{m}^{2}\right)$, adult $(\mathrm{M}=42.0 \pm 10.72$ years $)$ users $(82.4 \%$ female $)$ of an evidence-based, multidisciplinary Internet weight loss program was performed. Predictor variables included: PFS and PBLC, age, and longest maintained weight loss in relation to 1) BMI 2) TU.

Results: Both PBLC and PFS were correlated with baseline BMI and TU. Regression analyses indicated that only PFS independently predicted BMI $(\mathrm{p}=.0001)$ and $\mathrm{TU}(\mathrm{p}=.001)$ when the model included all predictor variables. One-way ANOVA indicated gender differences on both PBLC and PFS scores $(p=.001)$. Subsequent regression analyses separated by gender showed that in females PFS predicted BMI $(\mathrm{p}=.0001)$ and TU $(\mathrm{p}=.005)$. For males no variable significantly predicted BMI ( $p$ 's $>.05)$ however PBLC did predict TU $(p=.008)$.

Conclusions: Our findings suggest that when developing online weight loss programs clinical characteristics of the user could inform website algorithms to maximize website utilization. Gender differences indicated that for women it may be important to understand how factors related to the psychological influence of food impact utilization of online weight loss programs, however, for men broader barriers to lifestyle change is an important consideration.
\end{abstract}

Keywords: Adherence, information architecture, Internet, obesity, self-help, utilization, web-based, weight loss.

\section{INTRODUCTION}

Delivering health interventions direct-to-the-consumer via the Internet is increasingly recognized as a legitimate treatment modality with the capability for wide dissemination of evidence-based intervention to the general public and to populations traditionally disenfranchised from such intervention due to their distant proximity to medical and psychiatric services [1].

This modality is particularly relevant in the case of significant and widespread threats to public health such as obesity, since traditional clinic-based interventions are expensive to deliver and thus not widely available. Furthermore, in the area of weight management, the public is being bombarded with non-evidence-based and at times inappropriate interventions that are appealing to them due to the use of technologically advanced delivery systems. This has made examining the utility of the Internet for the delivery of reputable weight loss treatment on a large scale, using clinically meaningful assessment and intervention, an essential next step in the evolution of weight loss treatments. A key component of this evolution is the examination of these programs outside the highly controlled clinical trial setting. This is based, in part, on the fact that previous studies have indicated key differences in user behavior in this ad libitum environment where the user enrolls of their

*Address correspondence to this author at the Binks Behavioral Health PLLC, 2801 Canter Drive, Hillsborough, NC 27278, USA; Tel: (919) 7325524; Fax: (919) 383-5577; E-mail: mbinks@binksbehavioralhealth.com own accord via natural, public Internet search (not part of a study) and freely selects which program elements to use or not use as opposed to the less natural highly structured and monitored clinical trial settings [2,3].

With the majority of published Internet intervention research coming from controlled clinical trials [4], applied Internet programs face significant and unique challenges to broader dissemination and adherence [2,5] that extend beyond the typical threats to generalizability encountered when moving from the clinical trial to direct-to-consumer environments. This is due in part to the potential breadth of dissemination coupled with relatively low levels of contact with professionals to guide the treatment process. In addition, e-health programs are susceptible to poor utilization and attrition which limits their utility and effectiveness [6]. Combined, these factors make developing a complete understanding of the user, assessing important clinical information, and applying this to how they may or may not interact with the treatment essential. Furthermore, clinical trial based interventions rarely if ever examine the utilization patterns of the user beyond rather cursory details such as overall attrition rates, site logins and nutrition/weight tracking and do not typically consider the degree to which the therapeutic intervention (e.g. accessing assessments, educational content and support group) was delivered and the utilization patterns of the individual user $[3,7,8]$.

In this archival analysis we considered the psychological impact of living in food-abundant environments (Power of Food Scale; PFS) and also perceived barriers to lifestyle 
change (PBLC) as predictors of body mass index (BMI) and website tool utilization (TU) using data gathered from a free, evidence-based, anonymously utilized, direct-to-consumer weight loss program that includes nutrition, fitness and behavioral information, self-assessments, monitoring tools, and moderated support group message boards (social network). We hypothesized that higher PBLC and PFS scores would predict higher TU. The prediction is based on our belief derived from both our clinical experience and based in self-efficacy theory which suggests that behavior change is facilitated by a personal sense of control. If people believe that they can take action to solve a problem instrumentally, they are more likely to take action [9]. Our experience suggests that in obese populations, making the individual aware of issues relating to food and or barriers they face engenders greater effort to exert personal control by obtaining information and support; in this case utilization of online program elements. We also hypothesized that higher PFS and PBLC scores would predict BMI as each of these constructs (i.e. heightened vulnerability to the influence of the food environment and or higher perceived barriers to lifestyle change) may have likely resulted in less weight loss success in the past and consequently higher baseline BMI.

\section{MATERIALS AND METHODOLOGY}

\section{Setting \& Program Description}

The Healthy Weight Center [10] (HWC; http://www.healthyweightcenter.net), is a free access, anonymously utilized, evidence-based Internet weight loss program that provides nutrition, fitness and behavioral information, monitoring tools, interactive assessments with tailored feedback and moderated support group message boards (social networks). The intervention is based on review of design principles and consumer testing across multiple e-health platforms (i.e. smoking cessation, depression, panic disorder and problem drinking) and usability testing [11]. The program utilizes free-form-matrix design (all program elements available to every user) and also encourages full utilization through an online program guide.

\section{Study Design}

This study is a retrospective review of de-identified registration data obtained from Dec 2007 through Oct 2010 from users of HWC. All those who had complete registration data and who had completed the relationship with food exercise which included the Power of Food Scale (PFS) [12] and Perceived Barriers to Lifestyle Change (PBLC) [13] questionnaire were eligible for inclusion. There were no other exclusion criteria.

\section{Subjects}

An archival data analysis of all $(\mathrm{N}=1361)$ overweight/obese (BMI M $\left.=31.6 \pm 6.24 \mathrm{~kg} / \mathrm{m}^{2}\right)$, adult $(\mathrm{M}=$ $42.0 \pm 10.72$ years $)$ online program users $(82.4 \%$ female $)$ was undertaken. The program was available at no cost to all who have access to the World Wide Web from all geographic regions of the world. There was no advertising or promotion of the program. A link was added to the Evolution Health corporate site [14] and potential users could also encounter the program using publically available natural
Internet search. The program is available ongoing and this sample was drawn from all users of the program during the time period from Dec 2007 through Oct 2010. There was no formal recruitment for the program. To enroll, users completed an anonymous online registration process which included electronically endorsing the program disclosure agreement (informed consent) explaining that their deidentified data would be used for research purposes. Mean days participants remained actively enrolled in the program (registration date to last login) were: for the entire sample $65.9 \pm 123.16$ days; for males $70.65 \pm 124.74$ days; for females, $64.83 \pm 122.85$ days.

\section{Data Collection}

Data were extracted from the existing HWC database (archival data). All online surveys adhered to international privacy guidelines $[15,16]$. Procedures were in accordance with the Helsinki Declaration of 1975, as revised in 2008 [17]. Based on the use of anonymously collected, deidentified archival data the study met the requirements set forth in HIPPA- 45CFR164 section 514 (b) "HIPPA Privacy Rule" and is considered exempt.

\section{Personal Characteristics \& Behavior}

Data were gathered via online questionnaires and website analysis tools respectively. Body Mass Index (BMI) was based on self-reported height and weight $\left(\mathrm{kg} / \mathrm{m}^{2}\right)$. Patients were also asked to self-report the longest period they had ever maintained a weight loss of 10 pounds or greater (longest period of maintained weight loss; LPM).

\section{Website Utilization}

The variable Tool Utilization (TU) was calculated by counting the total number of times the user accessed each of the following program elements for the entire duration of their participation on the website: Meal planner, activity tracker, weight tracker, journaling tool, nutrition content lookup tool, and total number of posts to the moderated support group. This is a continuous variable ranging from zero to unlimited access (TU; Sample Range $=0$ - 989).

\section{Questionnaires}

Power of Food Scale (PFS) [12]: The PFS is a validated self-report inventory that assesses the psychological impact of living in food-abundant environments. It measures appetite for, rather than consumption of, palatable foods, at three levels of food proximity (food available, food present, and food tasted). The original 18 -item version was used in this study. Scoring involves summing of item ratings that are based on a 5-point Likert scale (1-5) for a total PFS score (higher scores indicate stronger influence of food cues). This version of the scale has not previously been tested in an online weight loss environment; however it has demonstrated reliability and validity in a previously published validation study administered in-person to obese subjects [12]. In this sample Cronbach's alpha indicated excellent internal consistency of the scale $(\alpha=.949)$.

Perceived Barriers to Lifestyle Change (PBLC) [13]: The PBLC questionnaire is a not yet validated 30 -item measure designed to tap commonly held beliefs about barriers to losing weight (e.g. I have a slow metabolism; My family members don't like to "eat healthy"). Scoring involves 
summing of item ratings that are based on a 4-point Likert scale (0-3) for a total PBLC score (higher scores indicate greater perceived barriers). Although the scale has not yet undergone formal validation, in this sample Cronbach's alpha indicated good internal consistency $(\alpha=.853)$.

\section{Data Analysis}

We considered the following predictor variables for the complete sample utilizing multiple linear regression analysis: PBLC, PFS, Age, and LPM in relation to 1) BMI 2) TU. One-way analysis of variance (ANOVA) was used to compare males and females on the PBLC and the PFS. Based on differences obtained, the sample was divided by gender and separate regression analyses were repeated using the same predictor models outlined above.

\section{RESULTS}

Table 1 summarizes the descriptive characteristics for variables of interest: Age, Weight, BMI, LPM, TU, PBLC and PFS for the whole sample and by gender. Pearson correlations for the entire sample $(\mathrm{N}=1361)$ indicated that PBLC was correlated with baseline BMI $(r=.080, p=.002)$. Similarly PBLC was correlated with TU $(r=.076, \mathrm{p}=.003)$. Pearson correlations also indicated that PFS was correlated with baseline BMI $(\mathrm{r}=.119, \mathrm{p}=.0001)$ and TU $(\mathrm{r}=.087, \mathrm{p}$ $=.001)$. Subsequent regression analyses indicated that only PFS (not PBLC) independently predicted BMI $(\mathrm{p}=.0001)$ and TU $(\mathrm{p}=.001)$ when the model included all predictor variables using backward regression procedures in the whole sample. Table 2 lists the final step of the backward regression procedure that initially included the following predictor variables in relation to BMI and TU: Age, LPM, PBLC, and PFS.

One-way ANOVA indicated gender differences on both $\operatorname{PBLC}(\mathrm{F}(1,1359)=6.72, \mathrm{p}=.01)$ and PFS $(\mathrm{F}(1,1359)=$ $6.497, \mathrm{p}=.01)$ total scores. Subsequent regression analyses separated by gender showed that in females PFS predicted BMI $(p=.0001)$ and TU $(p=.005)$. For males no variable significantly predicted BMI (p's > .05), however PBLC did predict TU $(\mathrm{p}=.008)$. Table 2 lists the final step of the backward regression procedure that initially included the following predictor variables in relation to $\mathrm{BMI}$ and $\mathrm{TU}$ : Age, LPM, PBLC, and PFS for each gender separately.

Table 1. Descriptive Characteristics of Sample

\begin{tabular}{|c|c|c|c|}
\hline & $\begin{array}{c}\text { Sample } \\
\mathbf{N}=\mathbf{1 3 6 1}\end{array}$ & $\begin{array}{c}\text { Males } \\
\mathbf{n}=\mathbf{2 4 0}\end{array}$ & $\begin{array}{c}\text { Females } \\
\mathbf{n = 1 1 2 1}\end{array}$ \\
\hline \hline Age (years) & $42.0 \pm 10.72$ & $41.54 \pm 10.81$ & $42.09 \pm 10.71$ \\
\hline Weight (kg) & $88.0 \pm 19.23$ & $98.74 \pm 18.16$ & $85.70 \pm 18.68$ \\
\hline LPM (months) & $17.35 \pm 49.68$ & $16.16 \pm 51.22$ & $17.61 \pm 49.37$ \\
\hline BMI (kg/m $\left.{ }^{2}\right)$ & $31.56 \pm 6.24$ & $31.04 \pm 5.18$ & $31.67 \pm 6.44$ \\
\hline TU & $6.60 \pm 39.99$ & $4.85 \pm 22.12$ & $6.98 \pm 42.86$ \\
\hline PBLC & $16.64 \pm 22.60$ & $13.22 \pm 19.49$ & $17.38 \pm 23.16$ \\
\hline PFS & $10.81 \pm 18.78$ & $8.01 \pm 16.07$ & $11.41 \pm 19.27$ \\
\hline
\end{tabular}

Table 2. Final Step of the Backward Regression

\begin{tabular}{|c|c|c|c|}
\hline & \multicolumn{2}{|c|}{ Unstandardized Coefficients } & \multirow{2}{*}{$\operatorname{Sig}(\mathbf{p})$} \\
\hline & B/Adjusted $\mathbf{R}^{2}$ & Std Error & \\
\hline \multicolumn{4}{|c|}{ BMI Sample } \\
\hline (Constant) & 31.136 & .194 & .0001 \\
\hline \multirow[t]{2}{*}{ PFS } & .039 & .009 & .0001 \\
\hline & $\mathrm{R}^{2}=.013$ & 6.20 & \\
\hline \multicolumn{4}{|l|}{ BMI Male } \\
\hline (Constant) & 28.831 & 1.325 & .0001 \\
\hline \multirow[t]{2}{*}{ Age } & .053 & .031 & .087 \\
\hline & $\mathrm{R}^{2}=.008$ & 5.16 & \\
\hline \multicolumn{4}{|c|}{ BMI Female } \\
\hline (Constant) & 31.190 & .222 & .0001 \\
\hline \multirow[t]{2}{*}{ PFS } & .042 & .10 & .0001 \\
\hline & $\mathrm{R}^{2}=.015$ & 6.39 & \\
\hline \multicolumn{4}{|l|}{ TU Sample } \\
\hline (Constant) & 4.60 & 1.246 & .0001 \\
\hline \multirow[t]{2}{*}{ PFS } & .185 & .058 & .001 \\
\hline & $\mathrm{R}^{2}=.007$ & 39.85 & \\
\hline \multicolumn{4}{|l|}{ TU Male } \\
\hline (Constant) & 2.293 & 1.705 & .180 \\
\hline \multirow[t]{2}{*}{ PBLC } & .194 & .073 & .008 \\
\hline & $\mathrm{R}^{2}=.025$ & 21.845 & \\
\hline \multicolumn{4}{|l|}{ TU Female } \\
\hline (Constant) & 4.831 & 1.483 & .001 \\
\hline \multirow[t]{2}{*}{ PFS } & .188 & .066 & .005 \\
\hline & $\mathrm{R}^{2}=.006$ & 42.720 & \\
\hline
\end{tabular}

\section{DISCUSSION}

This study highlights the importance of assessing commonly considered clinical parameters in ad libitum, direct-to-the-public online settings to identify users at risk for lower utilization of important Internet treatment program elements. The Power of Food Scale which measures the psychological impact of exposure to food cues in the environment showed a small yet significant relationship to baseline BMI which is consistent with available literature from other clinical populations suggesting that susceptibility to food cues is related to BMI in this sample. A similar relationship was seen for the PFS and website Tool Utilization suggesting that the PFS may provide information that can assist us in better understanding how users may utilize online weight loss programs. Further prospective and controlled data is needed to fully understand the strength and nature of this relationship. Perceived Barriers to Lifestyle Change, an as yet non-validated questionnaire that provides a face-valid assessment of commonly reported barriers to 
adherence to weight loss behaviors also showed comparable relationships to BMI and TU. Given the relatively large sample size and limited strength of the relationships observed, these data should be interpreted with caution. However they do suggest the potential for clinical measures such as these to provide insight into online behavior. These data represent a necessary first step in developing future studies to prospectively explore these and other relevant clinical predictors of website utilization if we are to develop a better understanding of how to improve Internet treatment utilization. Adherence to online interventions continues to be a significant clinical limitation of Internet treatments. Despite our knowledge of the utility of Internet interventions for weight loss in stand-alone clinical trials and in trials conducted in the primary care setting $[4,8]$; the issue of poor utilization continues to hamper our ability to use these tools effectively in efforts to reach people on a large scale with valid treatments [6].

Our results linking website utilization to specific clinical parameters in this naturalistically obtained, ad libitum, obese population of Internet treatment program users provides support for the notion that information gained through the initial registration process could be used to develop targeted content and strategies to enhance website utilization (and by extension treatment outcomes) based on the unique clinical characteristics of each individual user and achieved through technologically enhanced website algorithms. Conceptually this could mirror the process used in face-to-face clinical settings where information is gathered and clinical algorithms developed by the provider. In this case the algorithm for the individual would be created through the development of technology that attempts to attain maximal personalization targeting predefined user characteristics. This type of technology has long been used successfully in the online, direct-to-consumer product marketing arena and could be adapted for treatment tailoring based on the unique clinical characteristics of individual users. An example of these types of technical algorithms can be widely observed in online advertising, where advertisers target content to specific demographic and psychographic profiles. However, instead of utilizing this technology to sell goods and services, it could be utilized to increase individual relevance and optimize user engagement with treatment.

Clinically our findings also present some interesting information for consideration. Our hypothesis that higher PFS and PBLC would both lead to greater website utilization was supported for the PFS but not for PBLC as predictive models showed that only PFS was an independent predictor. This may have suggested a lack of utility for our measure of PBLC. However, further examination suggested that for men, higher PBLC predicted website utilization (and PFS did not); whereas for women the opposite was true with the PFS predicting their use of website tools. A similar overall pattern was seen for BMI in the whole sample and for women, with PFS predicting higher baseline BMI. For men however, neither PFS nor PBLC predicted baseline BMI.

It is difficult to explain the lack of relationship to baseline BMI in men in the context of the current study; however it is possible that for men neither PFS nor PBLC ultimately impact weight; whereas for women the direct influence of these factors on weight may be stronger. Nonetheless, taken as a whole, these findings support the need to consider men and women separately when examining the utility of online interventions for weight loss and when designing specific algorithms to enhance program engagement. Future studies should consider tailoring content in such a way as to take into account that for women factors related to the psychological influence of food may influence website utilization while broader barriers to lifestyle change may be more important considerations for male users. Further explorations of these phenomena using a wider array of validated instruments (and clinical parameters) and additional website tools (e.g. algorithm-based tailored text messaging) in relation to both website utilization and weight loss outcomes in direct-to-the-consumer weight loss programs are needed. Such efforts may provide insight into many of the as yet not well understood anomalies we see in how users access and utilize online treatment programs.

In addition to gender, future research should consider other subpopulations including racial and cultural identities in designing tailored assessments, website tools and educational content and in the development of automated treatment algorithms. Culturally relevant approaches to weight management are essential given the prevalence of obesity in African American and Hispanic and other underserved communities in both Canada and the United States [18-21]. If we are to design truly population-level interventions for weight management using advanced technological delivery systems we must attend to issues and content that are unique to these under-represented and underserved populations in ways that maximize relevance and in turn improve adherence to medical directives and promote positive treatment outcomes. Personal ethnic/cultural identity data is needed to understand the possible influences of these factors on Internet site utilization and to examine ways of tailoring content to culturally diverse populations. Some work has been done in the primary care setting which may be informative in developing interventions for wider dissemination to culturally diverse populations using these more naturalistic approaches [21,22].

Finally, while utilizing information gathered at assessment to inform clinical content is important, a successful approach to improving overall website utilization may also involve modifying information architecture (i.e. structural aspects of website design) [23]. Basing such modifications both on prior website user behavior patterns and also on clinical information obtained from the individual will allow Internet program developers to tailor design modifications that highlight access to the most personally relevant content and thereby increase program utilization, adherence and ultimately improve outcomes.

\section{Strengths and Limitations}

A notable strength of the present study is its generalizability to the typical experience of Internet users. Also of value is the ability of a program such as this to reach 
a wide audience due to the ease with which users can join and anonymously participate. The lack of available data relating treatment outcomes to utilization is also a limitation of the study. The use of a validated questionnaire in determining the psychological impact of living in foodabundant environments (PFS) on website utilization (TU) is strength. Overall low utilization rates may limit the generalizability of these findings, however these data may in fact be more representative of 'real-world' utilization of ad libitum Internet sites [3,23]. Further examination of sites of this type is warranted in order to determine what an appropriate baseline is in a naturalistic setting and to assess effective methods of improving utilization. Meta-analytic studies have suggested that higher usage of website features may be associated with positive weight change [24]. The relatively high completion rates of both the PBLC and PFS suggest that users are willing to participate in meaningful assessments online which represents a strength of this study. The relatively small amount of variability accounted for by the predictors in our study is a limitation. However we feel that at this early stage of research into broader factors impacting website utilization it is important to report these statistically significant findings to inform future research. In the broader obesity literature it has been previously noted that it is important to look beyond the major contributors to elevated obesity to include seemingly minor ones as these may have an additive effect [25]. The same may be true for contributors to online behavioral adherence.

\section{CONCLUSION}

To our knowledge, this is one of the first studies to provide insight into potential strategies for optimizing program design to improve utilization of core treatment elements. This is a necessary step to ensure quality when widely disseminating weight loss treatments on the Internet. Future studies should include detailed analysis of treatment delivery (i.e. utilization patterns) and how these may relate to both short- and long-term outcomes.

\section{ACKNOWLEDGEMENT}

Research relating to this abstract was funded by Evolution Health Systems Inc. Toronto, ON, Canada/San Francisco, CA, USA, the owner of the Healthy Weight Center software among other e-health platforms.

\section{CONFLICTS OF INTEREST}

Dr. Binks has acted as a paid consultant to Evolution Health Systems Inc. Toronto, ON, Canada/San Francisco, CA, USA, the owner of the Healthy Weight Center software among other e-health platforms. Trevor van Mierlo has a potential conflict of interest in the publication of this paper in that he is the CEO of Evolution Health Systems Inc.

\section{REFERENCES}

[1] Enwald HPK, Huotari MA. Preventing the obesity epidemic by second generation tailored health communication: an interdisciplinary review. J Med Internet Res 2010; 12(2):e24.

[2] Bonell C, Oakley A, Hargreaves J, Strange V, Rees R. Assessment of generalisability in trials of health interventions: suggested framework and systematic review. BMJ 2006; 333(7563):346-9.
[3] Binks M, van Mierlo T. Utilization patterns and user characteristics of an ad Libitum internet weight loss program. J Med Internet Res 2010; 12(1):e9.

[4] Kirbis-Weinstein PK. A review of weight loss programs delivered via the internet. J Cardiovasc Nurs 2006; 2(4):251-8.

[5] Cunningham JA, van Mierlo T. Methodological issues in the evaluation of internet-based interventions for problem drinking. Drug Alcohol Rev 2009; 28(1):12-17.

[6] Eysenbach G. The law of attrition. J Med Internet Res 2005; 7(1):e11.

[7] Jonasson J, Linne Y, Neovius M, Rössner S. An internet-based weight loss programme - a feasibility study with preliminary results from 4209 completers. Scand J Public Health 2009; 37(1):75-82.

[8] Bennett GG, Herring SG, Puleo E, Stein EK, Emmons KM, Gillman MW. Web-based weight loss in primary care: A randomized controlled trial. Obesity 2009; 18(2): 308-13.

[9] Bandura A. Self-efficacy mechanism in psychobiologic functioning. In: Schwarzer R, Ed. Self-efficacy: Thought control of action. Washington, DC: Hemisphere Publishing Corporation 1992; pp. 355-94.

[10] Healthy Weight Center. 2010; Available at: http://www.healthy weightcenter.net [Accessed 2010-02-01]. Archived at: $\mathrm{http}: / /$ www.webcitation.org $/ 5 \mathrm{nECOVnS3}$

[11] van Mierlo T, Cunningham J. Anonymous internet-based interventions for problem drinkers: design, development and dissemination. Abstract presented at the 35th Annual Alcohol Epidemiology Symposium of the Kettil Bruun Society, 2009, June; Copenhagen, Denmark. Available at: http://www.kbs2009.dk/ abstracts-kbs2009.pdf [Accessed 2010-02-01]. Archived at: http:// www.webcitation.org/5nEDcRziV

[12] Cappelleri JC, Bushmakin AG, Gerber RA, et al. Evaluating the power of food scale in obese subjects and a general sample of individuals: Development and measurement properties. Int J Obes 2009; 33(8): 913-22.

[13] Binks M, O'Neil PM, Perceived Barriers to Lifestyle Change (1999) Internally Developed Survey; Medical University of South Carolina, unpublished.

[14] Evolution Health Systems Inc. 2010; Available at http://www.evolutionhs.com/programs.aspx [Accessed 2010-0213]. Archived at: http://www.webcitation.org/5nW5plLtf

[15] Personal Information Protection and Electronic Documents Act (Canada). 2000 (amended 2004). Available at: http://www.priv.gc. ca/legislation/02_06_01_e.cfm [Accessed 2010-02-01]. Archived at: http://www.webcitation.org/5nEGDr6jp

[16] Health Insurance Portability and Accountability Act of 1996 (United States of America) (1996). Pub L No. 104-191. Available at: http://www.hhs.gov/ocr/office/index.html [Accessed: 2010-0201]. Archived at: http://www.webcitation.org/query?id=95708

[17] Ethical Principles for Medical Research Involving Human Subjects: Seventh revision. 59th WMA General Assembly, Seoul: October 2008. Available at: http://infomed.mds-ev.de/sindbad.nsf/ ddf5e481ed777582002567cb004313b9/75521880dcc4739dc12574 ee00376317/\$FILE/DoH_6Rev_SEOUL_2008-10.pdf [Accessed 2010-02-13] Archived at: http://www.webcitation.org/5nWFlChiw. Shields M, Carroll MD, Ogden CL. Adult obesity prevalence in Canada and the United States. NCHS data brief, no 56. Hyattsville, MD: National Center for Health Statistics. 2011.

[19] Flegal KM, Carroll MD, Cynthia LR, Ogden CL. Prevalence and trends in obesity among US adults 1999-2008, JAMA 2010; 303(3): 235-41.

[20] Tremblay MS, Perez CE, Ardem CI, Bryan SN, Katzmarzyk PT. Obesity, overweight and ethnicity Health Reports, Vol. 16, No. 4, June 2005 Statistics Canada, Catalogue 82-003

[21] Yeh HC, Clark JM, Emmons KE, et al. Independent but coordinated trials: insights from the practice-based Opportunities for Weight Reduction Trials Collaborative Research Group. Clin Trials 2010; 7: 322-32.

[22] Bennett GG, Herring SJ, Puleo E, Stein EK, Emmons KM, Gillman MW. Web-based weight loss in primary care: a randomized controlled trial. Obesity 2010;18,308-13.

[23] Danaher BG, McKay HG, Seeley JR. The information architecture of behavior change websites. J Med Internet Res 2005; 7(2):e12. 
[24] Neve M, Morgan PJ, Jones PR, Collins CE. Effectiveness of webbased interventions in achieving weight loss and weight loss maintenance in overweight and obese adults: a systematic review with meta-analysis. Obes Rev 2010;11(4): 306-21
[25] McAllister EJ, Dhurandhar NV, Keith SK, et al. Ten Putative Contributors to the Obesity Epidemic Crit Rev Food Sci Nutr 2009; 49(10): 868-913

Received: January 19, 2012

Revised: February 25, 2012

Accepted: March 5, 2012

(C) Binks et al.; Licensee Bentham Open.

This is an open access article licensed under the terms of the Creative Commons Attribution Non-Commercial License (http://creativecommons.org/licenses/by-nc /3.0/) which permits unrestricted, non-commercial use, distribution and reproduction in any medium, provided the work is properly cited. 